\title{
Research Article \\ Examining the Chaotic Behavior in Dynamical Systems by Means of the 0-1 Test
}

\author{
Loukas Zachilas and Iacovos N. Psarianos \\ Department of Economics, University of Thessaly, 43 Korai street, 38333 Volos, Greece \\ Correspondence should be addressed to Loukas Zachilas, zachilas@uth.gr
}

Received 23 January 2012; Revised 14 February 2012; Accepted 28 February 2012

Academic Editor: E. S. Van Vleck

Copyright (C) 2012 L. Zachilas and I. N. Psarianos. This is an open access article distributed under the Creative Commons Attribution License, which permits unrestricted use, distribution, and reproduction in any medium, provided the original work is properly cited.

We perform the stability analysis and we study the chaotic behavior of dynamical systems, which depict the 3-particle Toda lattice truncations through the lens of the 0-1 test, proposed by Gottwald and Melbourne. We prove that the new test applies successfully and with good accuracy in most of the cases we investigated. We perform some comparisons of the well-known maximum Lyapunov characteristic number method with the 0-1 method, and we claim that $0-1$ test can be subsidiary to the LCN method. The 0-1 test is a very efficient method for studying highly chaotic Hamiltonian systems of the kind we study in our paper and is particularly useful in characterizing the transition from regularity to chaos.

\section{Introduction}

The most popular technique for distinguishing between ordered and chaotic regions in Hamiltonian systems is to calculate Poincaré surface of section for various energies. We characterize the periodic orbit as stable or unstable by means of the Hénon's index [1]. However, the chaoticity of the nonperiodic orbits is estimated via the calculation of the maximum Lyapunov Characteristic Number (LCN). If $\mathrm{LCN}>0$, then we say that the orbit is chaotic, while if $\mathrm{LCN} \rightarrow 0$, the orbit is inside an island of closed invariants, hence is ordered.

Usually this technique requires quite a lot of computing time. In the case of a Hamiltonian system, we integrate two nearby orbits, and we compute the limit of the quantity, $\operatorname{LCN}(t)=\lim _{t \rightarrow \infty} \ln \left|\xi(t) / \xi\left(t_{0}\right)\right|$, where $\xi\left(t_{0}\right)$ and $\xi(t)$ are the distances between two points of the nearby orbits at times $t=0$ and $t$, respectively. This limit is the maximal Lyapunov Characteristic Number (LCN), while the quantity $\operatorname{LCN}(t)$ is known as the finite time LCN [2]. LCN measures essentially the average exponential deviation of two nearby orbits. For a definition, as well as for applications of LCN in other dynamical systems, see, for example, Contopoulos [3]. Later, Gao et al. [4, 5] introduced the scale-dependent Lyapunov 
exponent (SDLE) that can be accurately computed from short noisy time series and readily classify various types of motions.

In the present paper, we examine the chaotic behavior of the time-continuous truncated 3-particle Toda lattice through the lens of the "0-1 test", a method proposed by Gottwald and Melbourne [6]. This test distinguishes between order and chaos in deterministic systems and especially in cases at the edge of chaos and weak chaos. The central idea of the "0-1 test for chaos" is to distinguish the transition from an ordered to a chaotic region. The input is a time series of a relevant variable (if the equations of motion are known) or a time series of measurements (if the underlying equations are unknown). The output is 0 (zero) if the data correspond to motion in an ordered region or 1 (one) if the data correspond to motion in a chaotic region. Thus, our paper tries to contribute to the discussion by using this tool in a Hamiltonian that we have extensively studied its dynamical properties [7, 8].

The original method has been greatly improved recently $[9,10]$ and has been applied in several cases as in the van der Pol system, the driven and damped Kortweg-de Vries equation and an ecological model [6]; in Hénon-Heiles Hamiltonian system and Lorenz system [11]; in Ricker's population model [12]; in experimental data [13, 14]; in fluid dynamics [15]; in Hamiltonian dynamics [16, 17]; in a quasiperiodically forced system and strange nonchaotic attractors [18]; in nonsmooth mechanical systems [19, 20]. The method has been proven successful in most of these cases.

In Section 2, we briefly review the main steps of the 0-1 test. In Section 3, we present the 3-particle Toda lattice and its truncations. In Section 4, we compare the results of LCN $[7,8]$ with the results of the 0-1 test. In Section 5, we summarize the conclusions of this work.

\section{Description of the 0-1 Test for Chaos}

The method of the "0-1 test" starts with a data set $\varphi\left(t_{j}\right)$ for $j=1, \ldots, N$ (i.e., an observable of the underlying dynamics of finite length), sampled at discrete times $0<t_{1}<t_{2}<t_{3}<\cdots<$ $t_{N}$. In Hamiltonian dynamics, the sample data set is an orbit (i.e., a solution of the system, denoted by $\bar{x}$ ) and the length of the sampled set depends on the time step used in the RungeKutta algorithm of determination of the orbit and on the selection of $t_{j}, j \geq 1$. The truncated 3-particle Toda lattice is a 2D potential (see next section), and let $\bar{x}=(x, y, \dot{x}, \dot{y})$ be a solution of its equations of motion. We choose $\varphi=y$ and $t_{j}$ are the intersection times with a Poincaré cross section (i.e., $x=0$, see next section). So, the time series $\varphi\left(t_{j}\right)$ corresponds to observing a Poincaré map and thus we overpass any possible issues with oversampling [9]. In all our cases, we have integrated the equations of motion with a time step of $\Delta t=0.01$ and saved $N=100,000$ data points.

Then we introduce the following new coordinates:

$$
p(n)=\sum_{j=1}^{n} \varphi(j) \cos (j c), \quad q(n)=\sum_{j=1}^{n} \varphi(j) \sin (j c),
$$

where $n=1,2, \ldots, N$, and $c \in(0, \pi)[9,10,21]$.

Next, the diffusive (or nondiffusive) behavior of $p(n)$ and $q(n)$ is analyzed by calculating the mean square displacement $M(n)$ of one of the above new variables, say $p(n)$ :

$$
M(n)=\lim _{N \rightarrow \infty} \frac{1}{N} \sum_{j=1}^{N}[p(j+n)-p(j)]^{2}, \quad n=1,2,3, \ldots
$$


If the orbit is ordered, then $M(n)$ is a bounded function of $n$. If the orbit is chaotic, then $M(n)$ scales linearly with $n$ and the behavior of $p(n)$ is like a Brownian motion $[9,21]$.

Originally Gottwald and Melbourne $[6,21]$ tried to calculate the asymptotic growth rate via the linear regression method using $M(n)$. Later, they improved their own method introducing two major modifications. First, they replaced the mean square displacement $M(n)$ with the modified mean square displacement $D(n)$, where $D(n)=M(n)-V_{\text {osc }}(c, n)$. The need of the subtraction of the oscillatory term is the regularization of the linear behavior of $M(n)$ [9]. Second, they replaced the method of linear regression with the correlation method, which greatly outperforms the regression method in discrete dynamical systems.

Since in our paper all data sets in all cases we have studied, the observable data sets come from Poincaré cross section, we decided to apply the first method of linear regression and without subtracting any oscillatory terms. The possible need of improving our results will be investigated in a future paper by applying the correlation method.

Having calculated the mean square displacement $M(n)$ for $n=1,2, \ldots, N$, and since there was no oversampling, the next step was to estimate the asymptotic grown rate with the original method of linear regression.

In order to have an index of chaoticity, we define the asymptotic growth rate of the mean square displacement:

$$
K=\lim _{n \rightarrow \infty} \frac{\log M(n)}{\log n}
$$

Then, $K=0$ signifies regular dynamics (ordered motion), whereas $K=1$ signifies chaotic dynamics.

But since the data set has finite length, the mean square displacement (2.2) takes the following form:

$$
M(n)=\frac{1}{N-n} \sum_{n=1}^{N-n}[p(j+n)-p(j)]^{2}
$$

and the index $K$ is practically computed by performing a least-squares fit of $\log M(n)$ versus $\log n$, in the range $1 \leq n \leq N_{1}$ for a choice of $N_{1}$ such that $1 \ll N_{1} \ll N$ and $N_{1}=N / 10$. Although the observable is a Poincare map and there is no oversampling and in order to exclude any possible resonances between the frequencies of the Hamiltonian dynamical system and the arbitrary constant $c$, we performed all our computations of $K$ for 100 values of $c$ chosen randomly from the interval $(\pi / 5,4 \pi / 5)$ (instead of $(0, \pi)$, as it was suggested in [9]). The final value of $K$ is the median of the computed set $[9,21]$. Then, $K \approx 0$ stands for ordered motion and $K \approx 1$ signifies chaotic motion.

The new coordinates $p(n)$ and $q(n)$ in (2.1), along with $\vartheta(j)=j c$, can be viewed as a component of the solution to the skew product system:

$$
\begin{aligned}
& \vartheta(j+1)=\vartheta(j)+c, \\
& p(j+1)=p(j)+\varphi(j) \cos (\vartheta(j)), \\
& q(j+1)=q(j)+\varphi(j) \sin (\vartheta(j)),
\end{aligned}
$$


driven by the dynamics of the data set $\varphi(j), j=1, \ldots, N$. Here $(\vartheta, p, q)$ represents the coordinates on the Euclidean group of rotations $\vartheta$ and the translations $(p, q)$ in the plane [6]. It will be shown (Section 4) that the inspection of the dynamics of the trajectories on the $(p, q)$-plane provides a quick and simple visual test of whether the underlying dynamics is regular or chaotic.

\section{The Truncations of the 3-Particle Toda Lattice}

The Hamiltonian model we are studying is the special case of the periodic 3-particle Toda lattice first studied by Contopoulos and Polymilis [22]:

$$
H=\frac{1}{2}\left(q_{1}^{\prime 2}+q_{2}^{\prime 2}+q_{3}^{\prime 2}\right)+\exp \left(q_{1}-q_{2}\right)+\exp \left(q_{2}-q_{3}\right)+\exp \left(q_{3}-q_{1}\right)-3
$$

where $q_{i}, q_{i}^{\prime}(i=1,2,3)$ are the positions and momenta of the 3 particles, respectively. The general form of the Toda lattice [23] is a Hamiltonian for $N$ particles in a cyclic configuration. The Toda lattice can be generalized to infinite dimensions. In the limit, we reach the Korteweg-de Vries partial differential equation that has solitary wave solutions, called solitons [3].

Applying a canonical transformation, we can reduce the Hamiltonian, (3.1), to a 2dimensional system. If we expand the reduced Hamiltonian in series and truncate it at orders $3 \leq n \leq 20$, we get 18 new Hamiltonians [7, 8]. The most known is the third-order truncation, which coincides with the Hénon-Heiles system [24]. The 18 analytical functions are presented in the Appendix of Zachilas [7, 8].

The general form of the truncated Hamiltonians is

$$
H_{i}=\frac{1}{2}\left(x^{\prime 2}+y^{\prime 2}\right)+\Phi_{i}(x, y)=E,
$$

where $\Phi_{i}(x, y)$ is the potential function and $E$ is the energy integral $(i=3,4, \ldots, 20)$. The initial conditions to all orbits we calculated were chosen on the Poincare surface of section $x=0$ (i.e., the initial conditions have the form $\left(y, y^{\prime}, x^{\prime}=\sqrt{2\left(E-\Phi_{i}\right)-y^{\prime 2}}\right)$ for a given value of the energy $E$ and considering that $x^{\prime}>0$ ).

The 18 Hamiltonian systems are divided in two major categories. The odd-order ones (i.e., those that are truncated at odd order, for example, $n=3,5,7, \ldots, 19)$ and the evenorder systems (i.e., those that are truncated at even order, for example, $n=4,6,8, \ldots, 20$ ). The behavior and the characteristics of the two categories have some common features, but they also have a lot of differences $[7,8]$. The main differences are (i) the odd-order systems have escape energy, while the even-order ones do not, (ii) the onset of chaos in odd-order truncations comes via an unstable invariant point on the $y$-axis, while in even-order systems chaos comes via a couple of unstable invariant points on the $y^{\prime}$-axis, and (iii) the transition to chaos is not abrupt in even-order systems, while in odd-order ones is quite abrupt (mainly because of the escape energy).

Apart of the crucial differences, the two categories have a significant similarity, namely, that both are characterized by the presence of chaos. Extensive presentation of Poincaré surfaces of section and plots of $y$-coordinate versus LCN has been done in Zachilas $[7,8]$. 


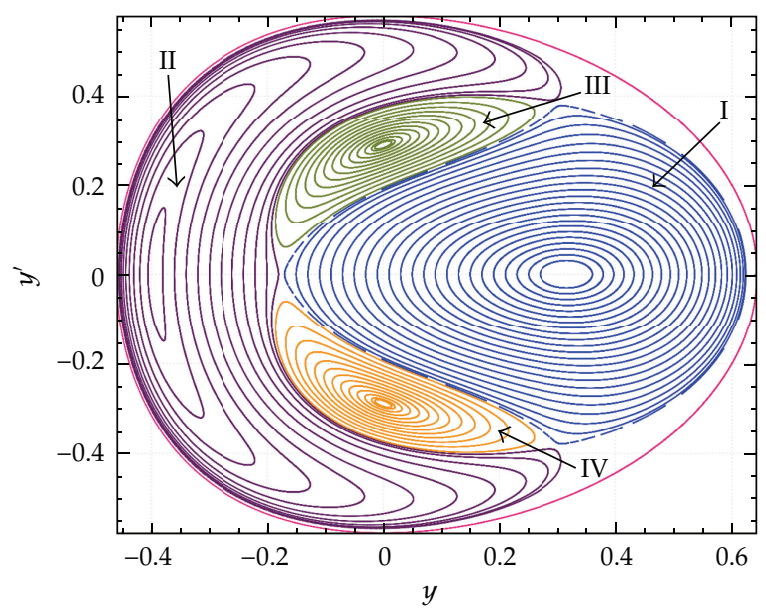

Figure 1: Poincaré surface of section at $E=0.166666$ (in fifth-order Hamiltonian). We observe four families of closed invariants: I (blue), II (purple), III (green), and IV (orange). The surrounding curve is the ZCV (Zero Velocity Curve).

\section{Tracing Chaotic Regions by Means of the 0-1 Test}

In order to distinguish the main points of interest and to cover all the range of truncations, we have selected two characteristic Hamiltonians of each category (one Hamiltonian with low-order and one with high-order truncations). In the odd-order category, we have chosen the fifth-order and the 19th-order Hamiltonians. In the even-order category, we have picked out the cases of sixth-order and 20th-order truncations. In Contopoulos [3] and Zachilas [7, 8] is proved that the Toda lattice Hamiltonians become less chaotic as we increase the order of truncations. This task had to be verified by means of the $0-1$ test.

\subsection{Odd-Order Hamiltonians}

The fifth-order truncation is a potential that has a lot of similarities with the Hénon-Heiles potential. The escape energy has the value of 0.3586468 (approximately the double with respect to the Hénon-Heiles one). In fifth-order truncation (like in all odd-order ones), we have found four families of closed invariant curves, plus one that surrounds all the curves and it is called Zero Velocity Curve (in Figure 1, ZVC is in pink). The Poincaré surface of section, at $E=0.166666$ (Figure 1), depicts these families, which are named: I (blue), II (purple), III (green), and IV (orange).

At this energy chaos does not appear [5] and all initial conditions give LCN $\rightarrow 0$. The corresponding behavior of an orbit (with initial conditions inside the closed invariant of family I) in the $(p, q)$-plane is depicted in Figure 2 . We can see that ordered motion corresponds to a bounded trajectory. The calculation of the mean square displacement in (2.3), gives $K=0$, which also characterizes the orbit as regular.

Chaos appears for $E=0.21$, as we can see in the Poincaré surface of section in Figure 3. It is introduced via the unstable invariant point $(U)\left(y \approx-0.21, y^{\prime}=0\right)$. The calculation of $\operatorname{LCN}$ (for $T=3 \times 10^{6}$ time units) gave the value about 0.0183 , which is a positive number, signifying that the orbit is a chaotic one. The corresponding motion on the $(p, q)$ plane is depicted in Figure 4. The figure shows a "fuzzy" shape. The chaotic motion, which is 


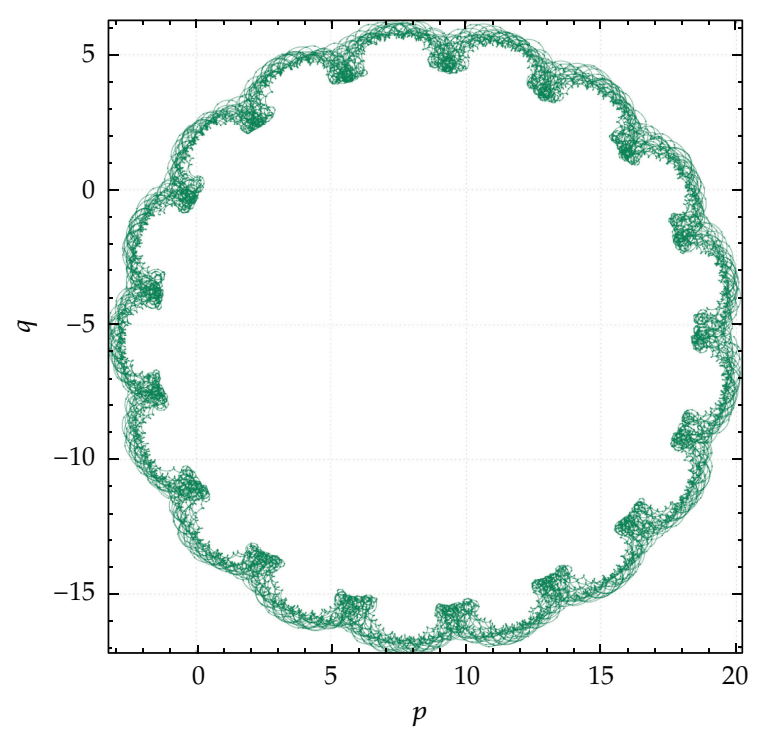

Figure 2: A regular orbit at $E=0.166666$ on the $(p, q)$-plane.

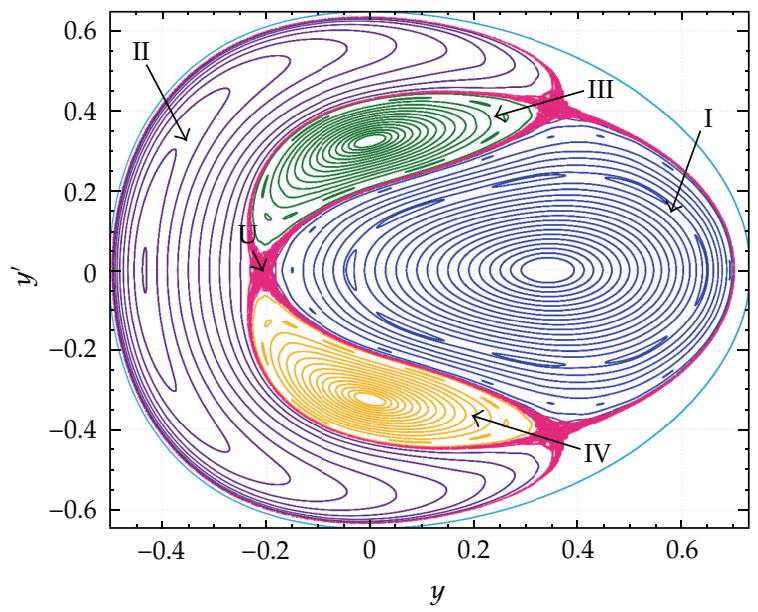

Figure 3: Poincaré surface of section at $E=0.21$ (in fifth order Hamiltonian). We can distinguish the four families (I, II, III, and IV) plus an unstable invariant point $(U)$, which characterizes the case as weakly chaotic.

also detected by the $0-1$ test, is well reflected by the unbounded Brownian-like diffusion. The presence of darker circle-shaped regions in Figure 4 indicates that inside the chaotic layer-that we observe among the islands of stability in Figure 3-we have the presence of cantori. As a consequence, we observe the phenomenon of stickiness [25]. We, also, observe that despite the fuzziness of the shape, the points in the $(p, q)$-plane occupy a box with approximate dimensions $(44 \times 77)$. The calculation of index $K$ gave, $K \approx 0.7558$, which is rather far from 1 (as the theory provides). The possible explanation of this value of $K$ might be that for $E=0.21$, chaos is not yet so evident and the case is characterized as weakly chaotic. The small value of $\mathrm{LCN} \approx 0.0183$ verifies, also, the result. It is then probable that the $0-1$ test 


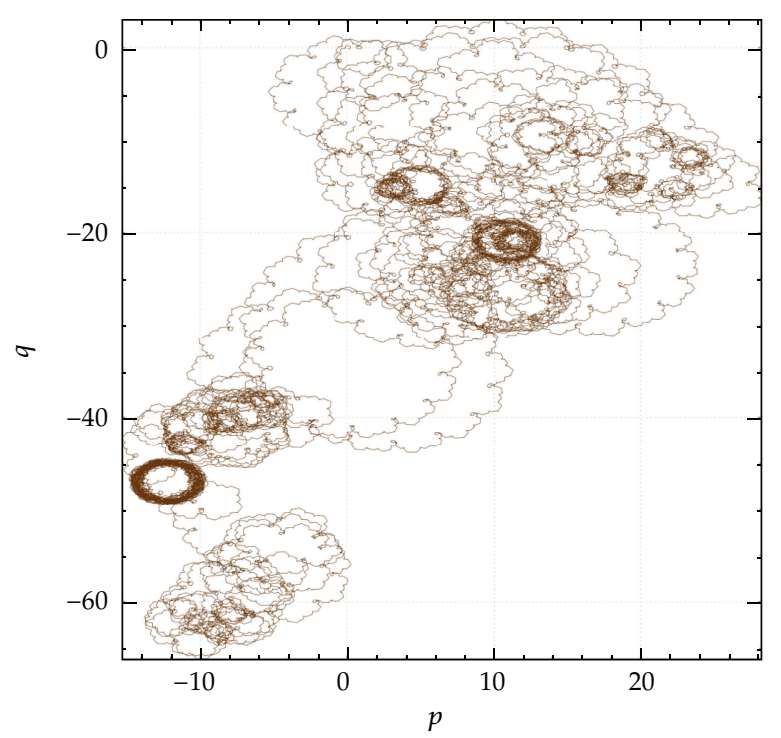

Figure 4: The weakly chaotic orbit at $E=0.21$ (in fifth-order Hamiltonian) on the $(p, q)$-plane.

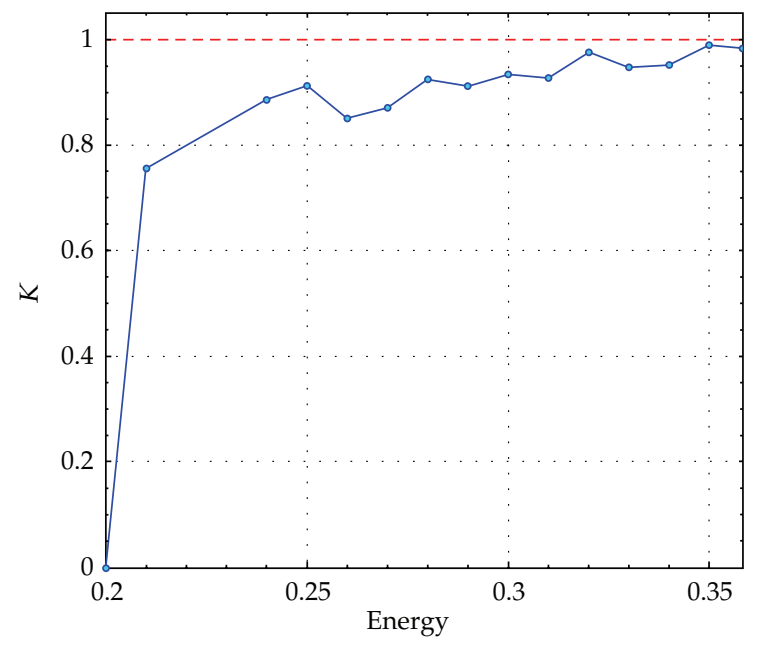

Figure 5: Index $K$ versus Energy in fifth-order Hamiltonian. The red dashed line corresponds to $K=1$.

cannot classify such cases not simply regular nor fully chaotic [14], and we have to reexamine it with the correlation method and/or subtracting oscillatory terms.

We have studied 15 cases (for $E=0.22$ up to escape energy $E=0.3586468$ ), taking initial conditions of strongly chaotic orbits. Figure 5 gives the successive values of index $K$ versus Energy. We can see that as we increase the energy $E$, the index $K$ increases too, tending to $1(K \rightarrow 1)$ (i.e., at $E=0.22, K \approx 0.7928$, at $E=0.24, K \approx 0.8863$, at $E=0.3, K \approx 0.9337$, while at the escape energy, $E=0.3586468, K \approx 0.9838$ ).

The 19th-order Hamiltonian is the most complicated odd-order one. It has 112 terms [7], where the last 10 terms are the 19th-order ones. The escape energy is much higher than in the fifth order, and now it is 13.08725629 . We have calculated and plotted quite a lot of orbits 


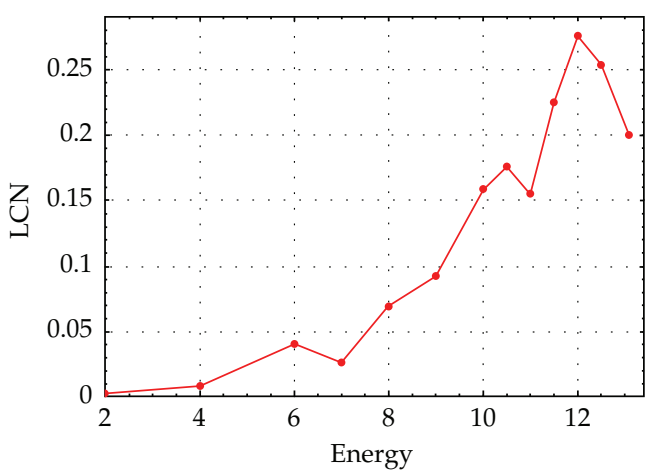

(a)

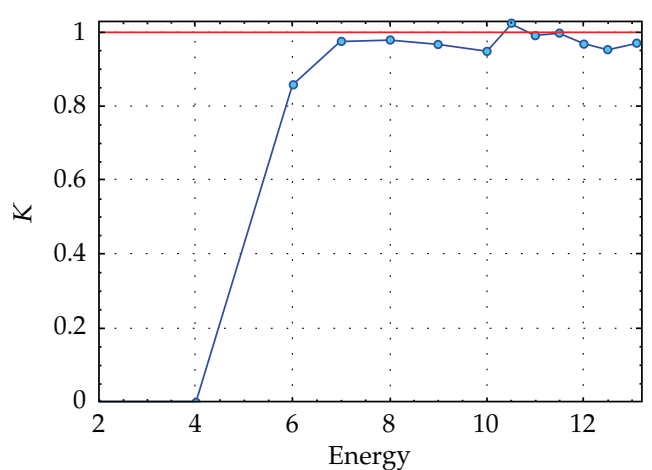

(b)

Figure 6: (a) LCN versus Energy in 19th-order Hamiltonian, (b) Index K versus Energy in the 19th-order Hamiltonian. There is conformity of both curves, but the onset of strong chaos appears abruptly at $E=6$ and is better shown in (b).

for $E=2$ up to $E=6$ with initial conditions inside closed invariants. In all tests, we observed that the points are bounded and constrained on ring-like shapes.

Chaos is absent at small energies and the first chaotic area appears at $E=6$. From $E=6$ up to escape energy $E=13.08725629$, the chaotic area increases considerably [7], but in all cases the Brownian-like motivo on the $(p, q)$-plane remains the same (more or less like the one in Figure 4). In Figures 6(a) and 6(b), we can observe the conformity in the evolution of the LCN and index $K$, respectively, versus Energy. Nevertheless, chaoticity is better shown in the diagram of index $K$ versus Energy, where at $E=6$ the index rises rapidly to 0.8575 and remains close to 1 for all energy values up to the escape energy.

\subsection{Even-Order Hamiltonians}

The sixth-order Hamiltonian, like all even-order Hamiltonians, has no escape energy, but contrary to odd-order ones, there are five main families of closed invariants, which are named [8] (Figure 7): I (blue), II (brown), III (purple), IV (green), and V (orange). On the axis $y=$ 0 (at $y^{\prime} \approx 0.14$ and $y^{\prime} \approx-0.14$ ), we can see two important families (VI and VII) of unstable invariants. The pink points belong to a single chaotic orbit. Using the initial conditions of this chaotic orbit, we get the corresponding plot on the $(p, q)$-plane, which is depicted in Figure 8.

We observe (as in Subsection 4.1. in odd-order Hamiltonians) the unbounded Brownian diffusion. At this small energy $(E=0.4)$, chaos is weak and that causes the motion to be trapped for a while in specific circle-shaped regions (i.e., the phenomenon of stickiness), but the general pattern is Brownian. Since there is no escape energy, there is no energy upper limit. In Zachilas [8], we have studied the sixth-order Hamiltonian up to the huge energy value of $E=5 \times 10^{6}$. In the present work, there was no need to explore again all those energies, and we just picked out some distinct energy values, so as to test the efficiency of 0-1 test.

We have calculated the Lyapunov Characteristic Number in a wide range of energies (Figure 9). If we pass over two energy values $(E=300$ and 500$)$, LCN increases rapidly from 0.03 (at $E=0.8$ ) to 1.03 (at $E=250$ ), while after this energy, LCN keeps increasing and it reaches the value of 1.267 (at $E=1000$ ). In all cases, the chaotic areas occupy important portions of the corresponding surface of section [8]. Small islands of closed invariants of oneperiodic families (I, III, II $a$, VI, VII), of double-periodic families $\left(\mathrm{IV}_{2 a}, \mathrm{~V}_{2 a}, \mathrm{VI}_{2 a}, \mathrm{VII}_{2 \mathrm{a}}\right)$, or even 




Figure 7: The Poincare section at $E=0.4$ (in sixth-order Hamiltonian). We distinguish five families of closed invariants: I (blue), II (brown), III (purple), IV (green), and V (orange). The unstable invariant points are marked as VI and VII, while the big pink area is a single chaotic orbit.

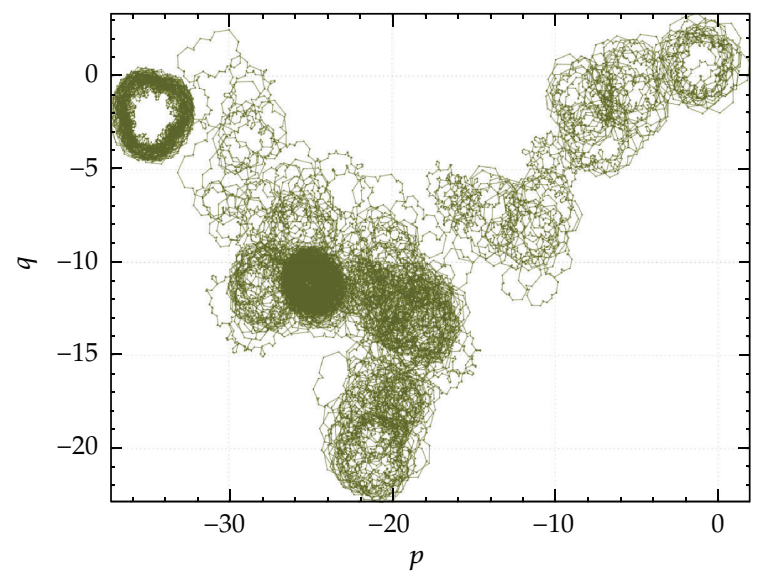

Figure 8: A regular orbit or a chaotic one? The calculation of index $K$ verifies the chaoticity.

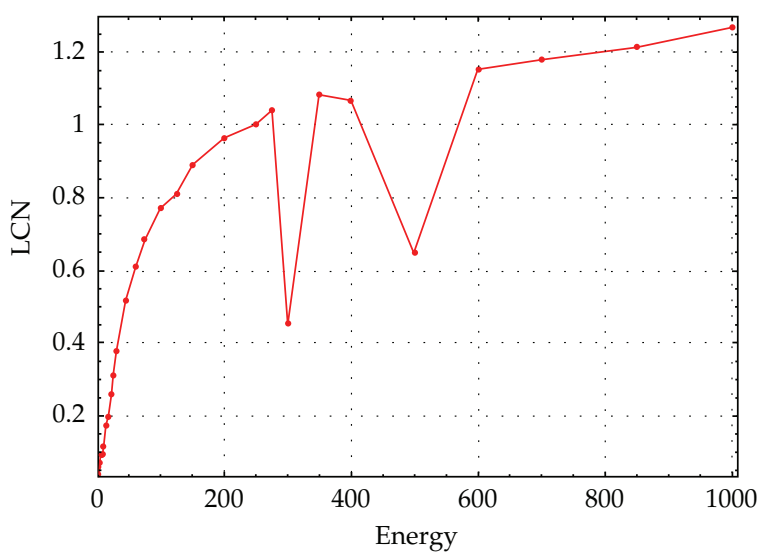

Figure 9: LCN versus Energy in sixth-order Hamiltonian. 


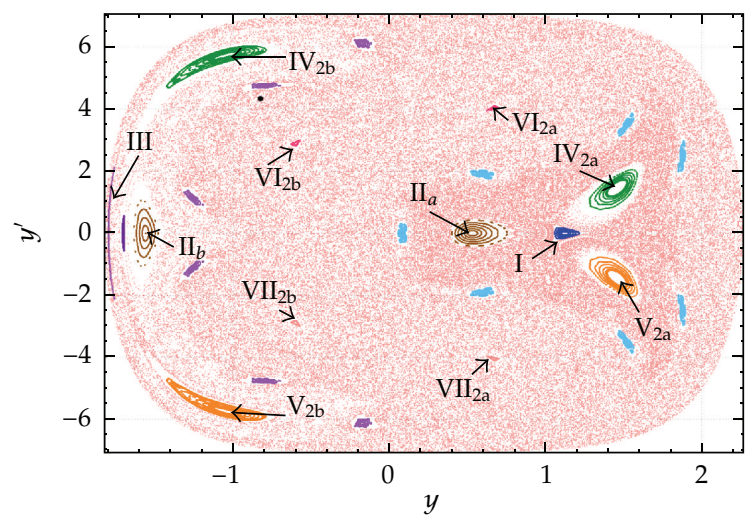

Figure 10: Poincaré surface of section at $E=18$ (in sixth-order Hamiltonian).

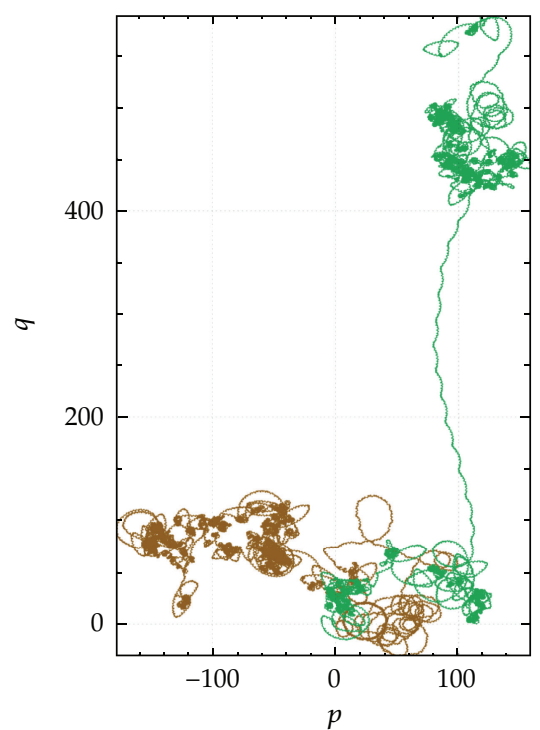

Figure 11: Representative chaotic orbits at the energy $E=18$ on the $(p, q)$-plane.

higher-periodic can be distinguished (Figure 10). We may, also, observe a small chaotic sea (in dark pink) surrounding families $\mathrm{I}, \mathrm{II}_{\mathrm{a}}, \mathrm{IV}_{2 \mathrm{a}}$, and $\mathrm{V}_{2 \mathrm{a}}$ embedded in the large chaotic sea (in pink).

Having used the same initial conditions, we have calculated and studied many orbits in various energies. The results were almost identical in all energies, except for $E=18$, where we have observed the two chaotic seas. We have taken initial conditions in both chaotic seas, and we have plotted two characteristic orbits (Figure 11). The one that has initial conditions inside the small sea is in brown colour, while the other in green colour has initial conditions inside the big sea. The second orbit diverged remarkably towards the $q$-direction (Figure 11). The pattern in all cases is the unbounded Brownian-like diffusion, which was previously observed in odd-order Hamiltonians. The plot ( $K$ versus Energy) in Figure 12 confirms the prediction. The index $K$ rises abruptly and at $E=45$, the calculation of index $K$ gave, $K \approx 1$, which signifies that chaos is evident and strong, even from small energy values. The index 


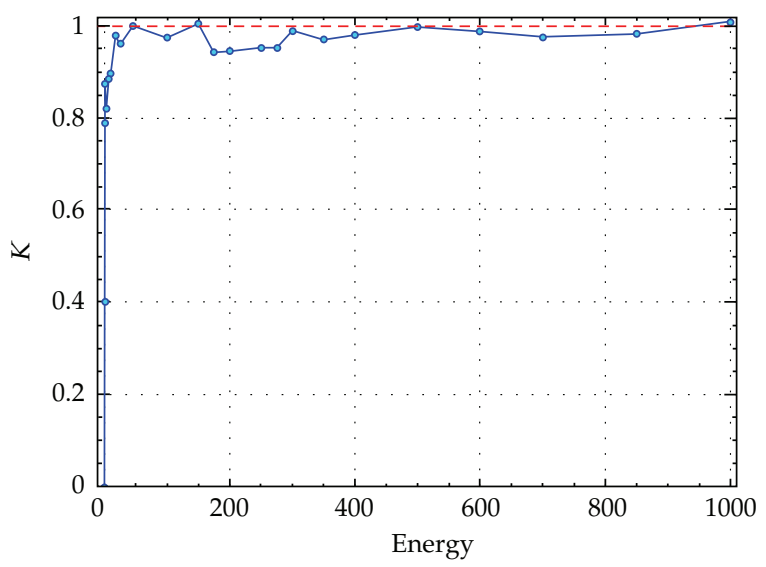

Figure 12: $K$ versus Energy in sixth-order Hamiltonian. The red dashed line corresponds to $K=1$.

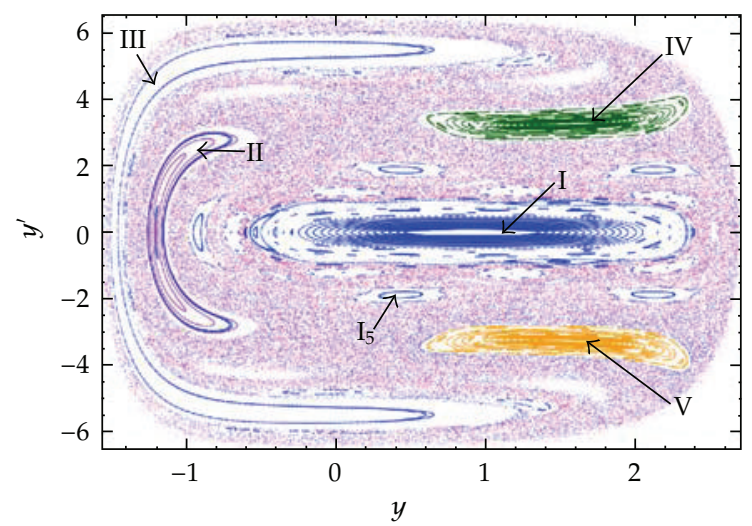

Figure 13: Poincaré surface of section at $E=20$ (in 20th-order Hamiltonian).

$K$ was calculated for the same range as in $(E, L C N)$ plot (Figure 9). We did not notice any peculiarities at $E=300$ and $E=500$, and the index remained close to one, certifying the chaotic behavior of the sixth-order Hamiltonian.

The last case for testing the reliability of the $0-1$ test is the 20th-order Hamiltonian. This was the most complicated Hamiltonian (among even and odd-order ones) with 123 terms. The first chaotic orbit appeared at $E=8$ at the unstable invariant points of Families VI and VII [8]. For $E<8$, the closed invariants do not permit the diffusion of the orbits [8] and the result is the bounded orbits on the $(p, q)$-plane.

For $E \geq 8$, chaos appears strikingly. We give the Poincaré surface of section at energy $E=20$ (Figure 13). The unbounded Brownian motion is depicted in Figure $14(E=20)$, where we plot two chaotic orbits (i.e., the "purple" orbit was calculated with initial conditions inside the cantorus of Family I and the "brown" orbit was calculated with initial conditions outside the cantorus). We can see that these orbits are close to each other in the beginning, but they deviate later, moving towards the lower right part of the plot (purple points) and the upper 


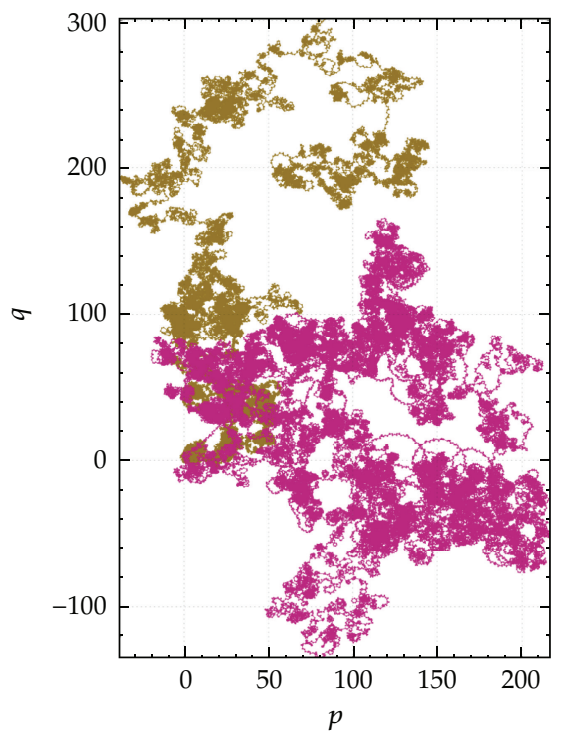

Figure 14: Unbounded Brownian-like orbits at $E=20$ (in 20th-order Hamiltonian). Two chaotic orbits with initial conditions inside and outside (in purple and brown, resp.) of the cantorus of Family I.

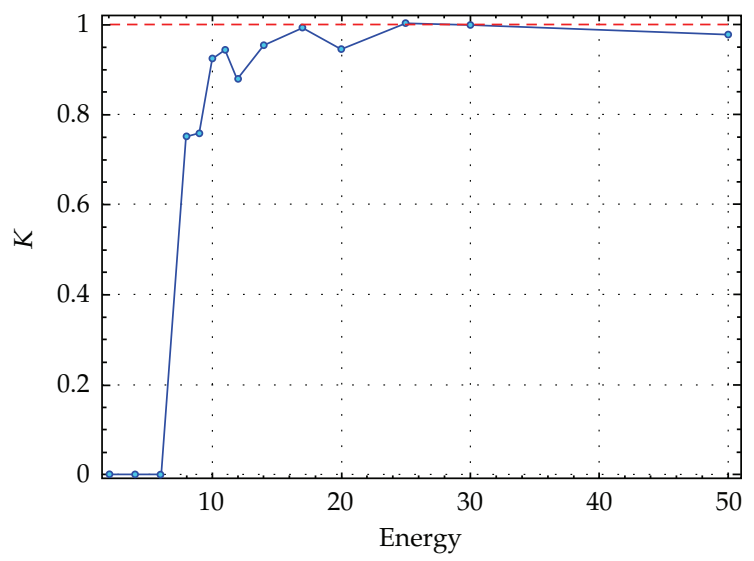

Figure 15: $K$ versus Energy in 20th-order Hamiltonian. The red dashed line is $K=1$.

part (brown points). The phenomenon of stickiness, which was previously observed in oddorder Hamiltonians, is rather obvious in this plot. The "purple" orbit creates more circleshaped regions than the "brown" orbit. The calculation of index $K$ resulted in $K \approx 0.9459$ for the "purple" orbit and $K \approx 0.9578$ for the "brown" one.

The global behavior of the 20th-order Hamiltonian is given in the diagram of Figure 15 ( $K$ versus energy). The index $K$ approaches the red dashed line $(K=1)$ almost from the beginning $(E=8)$ and remains close to 1 up to the end $(E=50)$. 


\section{Conclusions}

The 0-1 test provides a simple and reliable diagnostic criterion for the distinction of regular from strong chaotic orbits in Hamiltonian systems. We have worked the "0-1 test" in all 18 Hamiltonians $[7,8]$. In order to be as brief as possible, we have presented only four Hamiltonians (two odd-order truncations and two even-order ones). In all four cases, the 0-1 test proved to be mostly reliable. Every regular orbit has a bounded motion in the new coordinate system $(p, q)$. In some cases, calculating the LCN for regular or chaotic orbits helped. For regular orbits, LCN was always tending to zero, but quite a lot of times we had to integrate the equations of motion for millions of time units, in order to be sure that the orbit is a regular one. In contrast, the calculation of the asymptotic growth rate of the mean square displacement, index $K$, consumed less computing time [21] and the result for the regular orbits was always zero. Moreover, for the majority of the chaotic orbits in all Hamiltonians, the calculation of index $K$ confirmed that the orbits were indeed chaotic. The index $K$ was approximately 1 (or very close to 1 ), except for one or two cases of weak chaotic orbits, where we have to reexamine those cases by calculating the asymptotic growth rate with the correlation method and subtracting possible oscillatory terms.

In conclusion, our study shows that the $0-1$ test is a very useful method for examining the behavior of nonlinear Hamiltonian systems, taking into account that the index $K$

(a) is easily calculated easily and

(b) accurately detects the onset of strong chaos.

\section{References}

[1] M. Hénon, "Exploration numérique du problème restreint. II. Masses égales, stabilité des orbites périodiques," Annales d'Astrophysique, vol. 28, pp. 992-1007, 1965.

[2] Ch. Skokos, "The lyapunov characteristic exponents and their computation," Lecture Notes in Physics, vol. 790, pp. 63-135, 2010.

[3] G. Contopoulos, Order and Chaos in Dynamical Astronomy, Astronomy and Astrophysics Library, Springer, Berlin, Germany, 2002.

[4] J. B. Gao, J. Hu, W. W. Tung, and Y. H. Cao, "Distinguishing chaos from noise by scale-dependent Lyapunov exponent," Physical Review E, vol. 74, no. 6, Article ID 066204, 9 pages, 2006.

[5] J. B. Gao, Y. H. Cao, W. W. Tung, and J. Hu, Multiscale Analysis of Complex Time Series-Integration of Chaos and Random Fractal Theory, and Beyond, John Wiley \& Sons, Hoboken, NJ, USA, 2007.

[6] G. A. Gottwald and I. Melbourne, "A new test for chaos in deterministic systems," Proceedings of The Royal Society of London A, vol. 460, no. 2042, pp. 603-611, 2004.

[7] L. Zachilas, "A review study of the 3-particle Toda lattice and higher-order truncations: the odd-order cases (Part I)," International Journal of Bifurcation and Chaos in Applied Sciences and Engineering, vol. 20, no. 10, pp. 3007-3064, 2010.

[8] L. Zachilas, "A review study of the 3-particle Toda lattice and higher order truncations: the even-order cases (Part II)," International Journal of Bifurcation and Chaos in Applied Sciences and Engineering, vol. 20, no. 11, pp. 3391-3441, 2010.

[9] G. A. Gottwald and I. Melbourne, "On the implementation of the 0-1 test for chaos," SIAM Journal on Applied Dynamical Systems, vol. 8, no. 1, pp. 129-145, 2009.

[10] G. A. Gottwald and I. Melbourne, "On the validity of the 0-1 test for chaos," Nonlinearity, vol. 22, no. 6, pp. 1367-1382, 2009.

[11] J. D. Barrow and J. Levin, "A test of a test for chaos," http://arxiv.org/abs/nlin/0303070.

[12] A. Syta and G. Litak, "Stochastic description of the deterministic Ricker's population model," Chaos, Solitons and Fractals, vol. 37, no. 1, pp. 262-268, 2008.

[13] I. Falconer, G. A. Gottwald, I. Melbourne, and K. Wormnes, "Application of the 0-1 test for chaos to experimental data," SIAM Journal on Applied Dynamical Systems, vol. 6, no. 2, pp. 395-402, 2007.

[14] J. Hu, W. W. Tung, J. Gao, and Y. Cao, "Reliability of the 0-1 test for chaos," Physical Review E, vol. 72, no. 5, Article ID 056207, 5 pages, 2005. 
[15] N. Martinsen-Burrell, K. Julien, M. R. Petersen, and J. B. Weiss, "Merger and alignment in a reduced model for three-dimensional quasigeostrophic ellipsoidal vortices," Physics of Fluids, vol. 18, no. 5, Article ID 057101, 14 pages, 2006.

[16] M. Romero-Bastida, M. A. Olivares-Robles, and E. Braun, "Probing Hamiltonian dynamics by means of the 0-1 test for chaos," Journal of Physics A, vol. 42, no. 49, Article ID 495102, 17 pages, 2009.

[17] Ch. Skokos, Ch. Antonopoulos, T. C. Bountis, and M. N. Vrahatis, "Detecting order and chaos in Hamiltonian systems by the SALI method," Journal of Physics A, vol. 37, no. 24, pp. 6269-6284, 2004.

[18] J. H. P. Dawes and M. C. Freeland, "The 0-1 test for chaos and strange nonchaotic attractors," preprint.

[19] G. Litak, A. Syta, and M. Wiercigroch, "Identification of chaos in a cutting process by the 0-1 test," Chaos, Solitons and Fractals, vol. 40, no. 5, pp. 2095-2101, 2009.

[20] G. Litak, A. Syta, M. Budhraja, and I. M. Saha, “Detection of the chaotic behaviour of a bouncing ball by the 0-1 test," Chaos, Solitons and Fractals, vol. 42, no. 3, pp. 1511-1517, 2009.

[21] G. A. Gottwald and I. Melbourne, "Testing for chaos in deterministic systems with noise," Physica D. Nonlinear Phenomena, vol. 212, no. 1-2, pp. 100-110, 2005.

[22] G. Contopoulos and C. Polymilis, "Approximations of the 3-particle Toda lattice," Physica D, vol. 24, no. $1-3$, pp. 328-342, 1987.

[23] M. Toda, “Waves in nonlinear lattice," Progress of Theoretical Physics Supplement, vol. 45, p. 174, 1970.

[24] M. Hénon and C. Heiles, "The applicability of the third integral of motion: some numerical experiments," The Astronomical Journal, vol. 69, pp. 73-79, 1964.

[25] G. Contopoulos and M. Harsoula, "Stickiness effects in conservative systems," International Journal of Bifurcation and Chaos in Applied Sciences and Engineering, vol. 20, no. 7, pp. 2005-2043, 2010. 


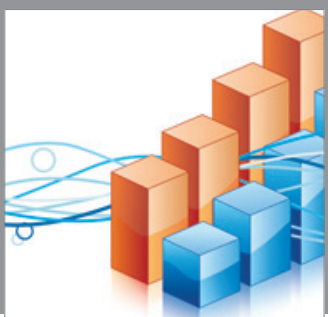

Advances in

Operations Research

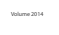

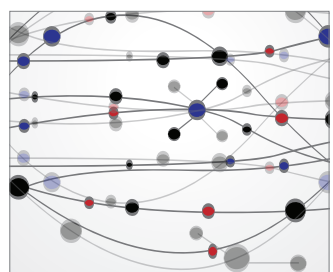

\section{The Scientific} World Journal
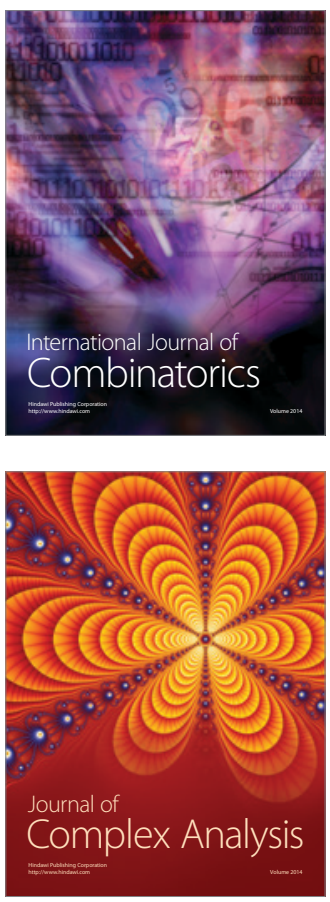

International Journal of

Mathematics and

Mathematical

Sciences
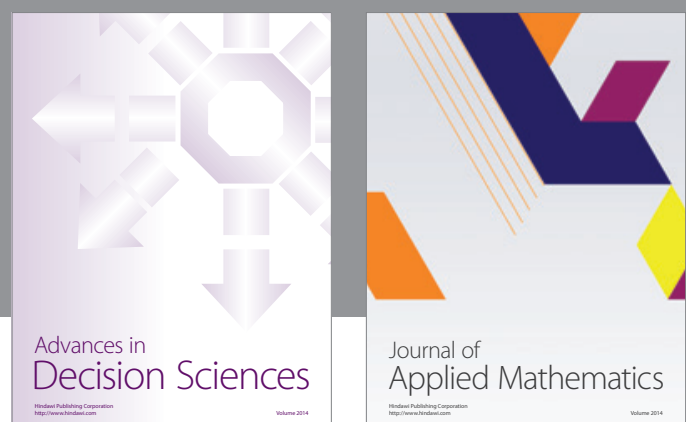

Journal of

Applied Mathematics
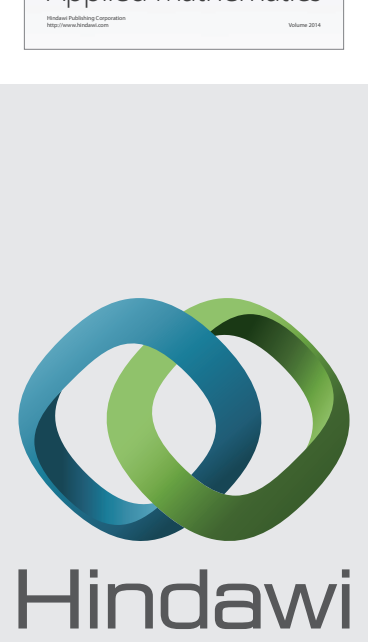

Submit your manuscripts at http://www.hindawi.com
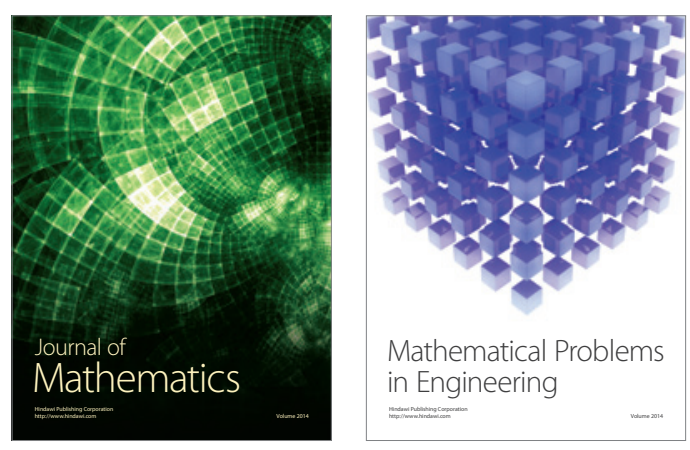

Mathematical Problems in Engineering
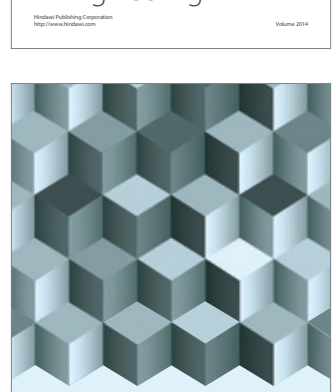

Journal of

Function Spaces
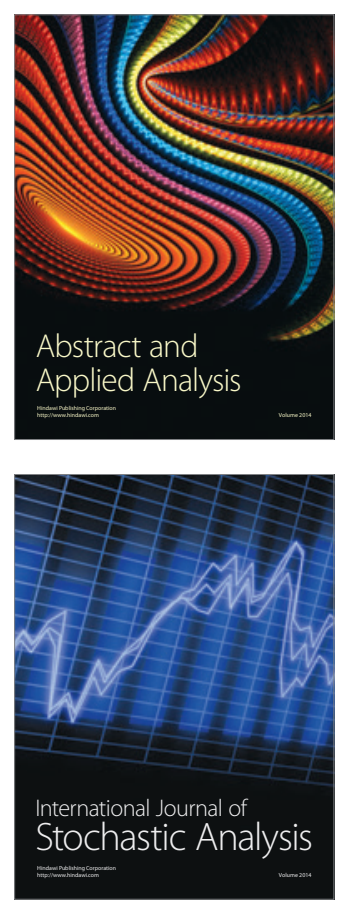

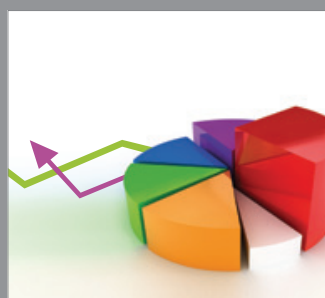

ournal of

Probability and Statistics

Promensencen
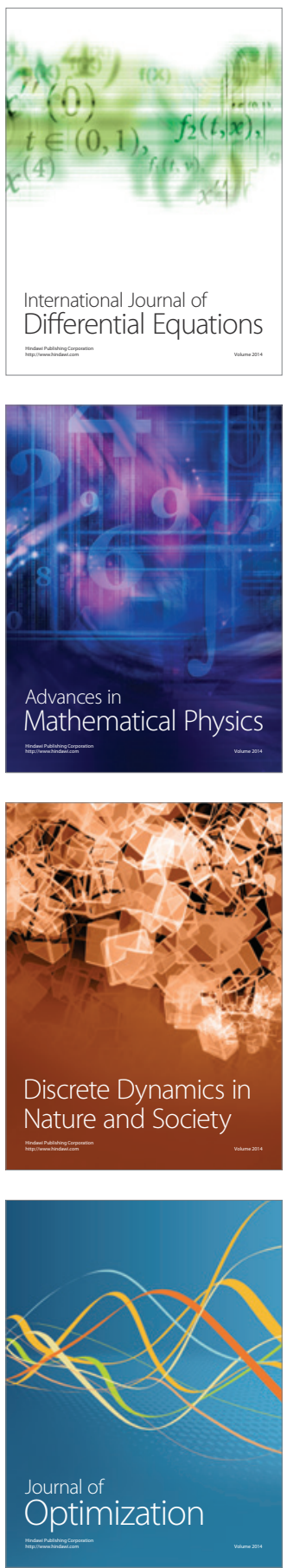